\section{Sostenibilità e moda: una prospettiva italiana}

Ida Palombella ${ }^{(1)}$

Resumen: La pandemia actual está cambiando la atención de los consumidores hacia la adopción de una actitud más sostenible en sus elecciones de consumo. A falta de una regulación nacional e internacional específica sobre sostenibilidad, a menudo son las mismas empresas o asociaciones de empresas, como la Cámara Nacional de Moda de Italia, las que adoptan autorregulaciones para garantizar el desarrollo de un modelo de negocio más sostenible. En este sentido, la propiedad intelectual es clave para distinguir un negocio sostenible, por un lado para las actividades de investigación y desarrollo que conducen a la presentación de patentes verdes; por otro, a través de la identificación de características sostenibles de productos y servicios identificados por marcas de certificación.

Palabras clave: sustentable - consumo - modelo de negocio - patentes verdes - marcas de certificación - propiedad intelectual.

[Resúmenes en inglés y portugués en las páginas 170-171]

(1) Especialista italiana que durante cuatro años seguidos, 2017, 2018, 2019 y 2020, fue elegida Abogada del año de Derecho de la Moda en Italia. Además ha sido rankeada por Chambers \& Partners Directory en el Top 500 de los abogados especialistas en Propiedad Intelectual del Sector de la Moda y el Lujo. Ida colabora activamente con la Camera Nazionale della Moda Italiana donde es miembro de la Comisión de Propiedad Intelectual y Sustenabilidad. Es jefe del Departamento de Propiedad Intelectual de Deloitte Italia y lider global de Deloitte TMT. Desde febrero 2021 es profesora en la Università Cattolica del Sacro Cuore de Milán en el nuevo curso universitario internacional de Fashion Law. ipalombella@deloitte.it

Nota de la Autora: si ringraziano Valentina Favero e Erica Saltalamacchia per la collaborazione nella stesura del presente articolo. 


\section{Introduzione}

L'emergenza sanitaria legata alla pandemia di COVID-19 insieme all'implementazione dei lockdown ha portato con sé anche l'inevitabile conseguenza della crisi economica e sociale nella maggior parte dei Paesi del mondo. Molte imprese si sono viste costrette, da un giorno all'altro, ad arrestarsi o a rallentare significativamente la propria attività produttiva $\mathrm{e}$ commerciale, senza nemmeno la possibilità di poter pianificare la ripresa delle attività ad un orizzonte certo.

Tra i settori che hanno subito il maggiore impatto di questa crisi, vi è sicuramente quello della moda che fin dalle prime settimane di lockdown ha rilevato tutti i limiti del proprio modello di business, in particolare quello fondato sul "fast fashion". Oltre alle problematiche già conosciute, quali gli eccessivi scarti di produzione e la gestione dello smaltimento dei rifiuti, legati a un'abbondanza di offerta, ha senz'altro impattato sul settore anche una supply chain fortemente globalizzata. Le varie fasi di realizzazione della produzione sono infatti dislocate in paesi molto lontani tra loro, con una grande concentrazione nel sud est asiatico, il che ha reso ancora più difficile la gestione immediata della crisi.

Il COVID-19 ha quindi messo in evidenza i limiti di un sistema che già da anni era oggetto di ampie critiche e discussioni, ovvero di un sistema fondato su processi produttivi che non possono purtroppo essere definiti, in alcun modo, "sostenibili".

Secondo degli studi condotti dall'Unione Europea, l'industria della moda, che rappresenta circa il 3\% del prodotto interno lordo della UE (Comisión Europea, s.f.) $)^{1}$, ha un impatto ambientale estremamente significativo. Basti considerare il quantitativo di risorse idriche necessario per produrre i tessuti come per coltivare il cotone e le altre fibre. Ad esempio, per la produzione di una semplice t-shirt si stima essere necessari 2.700 litri d'acqua, pari al fabbisogno di una persona per due anni e mezzo. Complessivamente, è stato stimato che solo nel 2015 l'industria tessile e dell'abbigliamento ha impiegato oltre 79 miliardi di metri cubi d'acqua, pari a quasi il 30\% del totale del fabbisogno idrico della UE. Ugualmente, si stima che la produzione tessile sia responsabile di circa il $20 \%$ dell'inquinamento globale delle acque pulite causato dai prodotti di tintura e finissaggio ed il $35 \%$ del totale delle microplastiche rilasciate nell'acqua degli oceani (Parlamento Europeo, 2021).

Ma non solo. L'impatto del settore non è solo ambientale, ma anche economico e sociale. A causa delle frequenti delocalizzazioni delle produzioni in aree geografiche caratterizzate da una insufficiente sicurezza del lavoro, non sono rare, purtroppo, le notizie relative agli sfruttamenti dei lavoratori e ad incidenti, anche gravissimi, nelle fabbriche off-shore. $\grave{\mathrm{E}}$ ancora fresca la memoria di uno dei più gravi incidenti verificatesi nel 2013 a Dacca in Bangladesh, dove l'edificio Rana Plaza, in cui erano presenti numerose fabbriche di abbigliamento che producevano capi per importanti marchi occidentali, crollò provocando la morte di 1.134 persone (Independient, 2020).

L'emergenza sanitaria in corso, ha quindi nuovamente stimolato la necessità di ripensamento del business model per le aziende del settore della moda, ispirandosi a modelli più sostenibili e riaccendendo il dibattito su un tema che oggi appare non più eludibile.

Il concetto di sviluppo sostenibile non è un concetto nuovo. È stato infatti introdotto nel 1987 nel Rapporto Brundtland (conosciuto anche come Our Common Future), pubblicato dalla Commissione mondiale su Ambiente e Sviluppo (WCED), attraverso cui si è 
tentato per la prima volta a livello internazionale di formulare delle linee guida volte a rendere compatibile la tutela dell'ambiente e lo sviluppo economico. Secondo il rapporto, lo sviluppo sostenibile è definito come: "lo sviluppo che è in grado di soddisfare i bisogni della generazione presente, senza compromettere la possibilità che le generazioni future riescano a soddisfare i propri" (World Comission, 1987).

Il rapporto stabiliva che le criticità dell'ambiente fossero causate dall'estrema povertà di una parte del mondo e dall'implementazione di modelli di produzione e di consumo non sostenibili nell'altra parte del mondo. Il rapporto evidenzia quindi la necessità di attuare una strategia in grado di conciliare le esigenze delle logiche di mercato con quelle ambientali e sociali, ovvero uno "sviluppo sostenibile".

In sostanza, implementare un modello ispirato ai principi di sviluppo sostenibile per un'azienda significa adottare una strategia di business che rispetti non solo l'ambiente e le sue risorse ma anche il benessere e la qualità della vita di tutti gli stakeholder, dai lavoratori fino ai consumatori finali. La sostenibilità è rappresentata quindi dal raggiungimento di un equilibrio tra il perseguimento della crescita economica dell'azienda e la tutela delle esigenze sociali e ambientali, ottenibile solo attraverso l'implementazione di determinate condotte.

Con specifico riferimento al settore della moda, l'approccio alla sostenibilità impatta sull'intera supply chain, coinvolgendo tutti gli elementi della catena di valore, partendo dalla progettazione ed il design fino ad arrivare alla distribuzione finale e al modello di fruizione del bene stesso.

Sotto questo aspetto, alla normale ricerca di efficienza che ciascuna azienda persegue nel corso della propria attività, lo scopo dovrebbe essere costituito dall'integrazione nel proprio core business di obiettivi strategici legati a:

- efficienza energetica, idrica e di tutte le risorse non rinnovabili;

- minori sprechi di materiali;

- riduzione dei costi della non sostenibilità (e non solo quelli derivanti da imposizioni normative);

- capacità di innovazione nello sviluppo di prodotti sostenibili;

- maggiore attenzione e trasparenza informativa nei rapporti con le comunità locali e con i clienti intermedi ed i consumatori finali (Tartaglione y otros, 2020).

A 30 anni dalla pubblicazione del Rapporto Brundtland, il concetto di sviluppo sostenibile non ha ancora trovato una sua vera e propria trasposizione normativa. Così, in mancanza di specifici vincoli o regole che spingano le imprese ad una maggiore sostenibilità, sono i singoli operatori del mercato, anche in forma associativa, a darsi delle norme di condotta e dei principi etici al fine di avvicinarsi sempre di più ad un modello di business più attento alle esigenze ambientali e sociali. 


\section{I tre pilastri dello sviluppo sostenibile}

In Italia, il settore della moda si è dato delle linee guida in ambito di sostenibilità attraverso il Manifesto della Sostenibilità (il "Manifesto") (Camera, 2012) promosso e pubblicato dalla Camera Nazionale della Moda Italiana ("CNMI")2. Il Manifesto, anche se rivolto innanzitutto alle imprese associate a CNMI, rappresenta un vero e proprio punto di riferimento per tutte le imprese italiane che producono e commercializzano prodotti di lusso nel mondo. Il decalogo definisce gli orientamenti ai quali tutti gli operatori della filiera dovrebbero ispirarsi al fine di realizzare dei business sempre più sostenibili. Il documento è suddiviso nelle diverse fasi della catena del valore, a cui si aggiungono alcuni principi trasversali. In particolare, si propone come obiettivo quello di guidare le imprese italiane verso la realizzazione di un modello che ponga maggiore attenzione agli aspetti ambientali e sociali, al contempo assistendo le imprese ad apprendere a gestire meglio i rischi di reputazione e operativi che possono derivare da una mancata attuazione di una strategia sostenibile. I dieci principi sottolineati dal Manifesto possono essere sostanzialmente ricondotti ai tre pilastri della sostenibilità:

1. la sostenibilità ambientale, ovvero la capacità di garantire nel tempo la disponibilità $\mathrm{e}$ la qualità delle risorse naturali;

2. la sostenibilità sociale, ovvero la capacità di garantire la qualità di vita e il benessere delle persone;

3. la sostenibilità economica, ovvero la capacità di garantire efficienza economica e produttiva per le imprese nel lungo termine.

Da una lettura del Manifesto, risulta evidente come questi tre pilastri siano indissolubilmente legati fra di loro e non sia possibile prescindere da uno di essi, senza che non ci siano conseguenze sugli altri.

Ad esempio, l'enfasi posta sulla sostenibilità ambientale sta spronando le aziende verso la ricerca di prodotti e di tecnologie e processi produttivi sempre maggiormente rispettosi dell'eco-sistema. Tuttavia, questa ricerca di innovazione, spesso comporta un aggravio dei costi produttivi che, se non si tradurranno in crescita del valore per il consumatore, rischieranno di riflettersi negativamente sulla stessa sostenibilità economica e sociale. È quindi ugualmente necessaria un'evoluzione culturale del modello di consumo, che conduca il cittadino-consumatore ad una migliore consapevolezza e sensibilità verso queste problematiche.

Considerando, quindi, i principi contenuti nel Manifesto attraverso la loro categorizzazione secondo i tre "pilastri della sostenibilità", è interessante approfondirne alcuni degli aspetti più attuali e rilevanti per l'Italia.

\section{Sostenibilità ambientale}

Come abbiamo visto, l'industria tessile impiega principalmente risorse non rinnovabili -circa 98 milioni di tonnellate all'anno-includendo anche il petrolio per la realizzazione 
di fibre sintetiche, i fertilizzanti per la coltivazione del cotone e le sostanze chimiche per la realizzazione delle tinture e i tessuti (Ellen, 2017). Inoltre, il sistema moda è attualmente caratterizzato da un limitato uso nel tempo dei materiali da parte dei consumatori (con conseguente necessario elevato livello di produttività) e da bassi livelli di riciclo (Ellen, 2017) (il modello "take, make and waste").

L'attuale ciclo produttivo dell'industria, non solo impatta direttamente sul problema della scarsità delle risorse naturali che utilizza estensivamente, ma anche in termini di impatto sulla problematica del "Climate Change", in quanto se l'attuale trend di crescita dell'industria della moda dovesse proseguire con questi livelli di produttività (al netto della battuta di arresto dovuta all'attuale pandemia), entro il 2050 assorbirebbe oltre il $26 \%$ del bilancio totale di $\mathrm{CO}_{2}$ associato alla necessità di limitare la crescita del riscaldamento globale a non oltre $2^{\circ}$ centigradi (Ellen, 2017).

Appare quindi necessario che, quando si parla di sostenibilità ambientale nel settore della moda, si tratti sostanzialmente della importanza di puntare sempre di più ad un'economia circolare fondata sui principi della riduzione dei rifiuti industriali, la riduzione dei consumi idrici, dell'allungamento del ciclo di vita dei prodotti e dei materiali in uso e il loro recupero e riciclo.

Ripensare il ciclo produttivo ed il relativo modello di consumo, in questo tipo di sistema, permetterebbe di immaginare un mondo dove i prodotti e i materiali mantengano un loro valore economico e funzionale allungandone la loro vita d'uso, senza divenire mai dei rifiuti "inutili".

Proprio in questo senso, il Manifesto, sottolinea in più punti le condotte che le aziende dovrebbero perseguire al fine di realizzare un modello di economia circolare.

Alcuni esempi di strategie o azioni sostenibili sono:

- integrare l'eco-compatibilità dei prodotti nella stessa progettazione e nel design, privilegiando la qualità del prodotto che ne aumenti la durata nel tempo, anche attraverso l'utilizzo di materiali a basso impatto ambientale e la riduzione degli scarti e dei rifiuti.

- Innovare i prodotti attraverso l'utilizzo di materiali riciclati e rigenerati, sperimentando anche nuovi modi per reinterpretare prodotti e materiali arrivati a fine ciclo di vita.

- Implementare processi produttivi caratterizzati dalla minimizzazione dei consumi delle risorse energetiche e naturali e/o dall'uso di sostanze chimiche nocive.

- Investire nella ricerca e nell'innovazione tecnologica.

- Sviluppare sistemi di gestione aziendale che permettano di controllare, misurare e verificare periodicamente la corretta implementazione delle strategie sostenibili.

- Assicurare l'allineamento della intera supply chain ai medesimi principi.

La realizzazione di questi obiettivi di sostenibilità ambientale passa quindi innanzitutto dall'impresa e dalla sua capacità di innovarsi, anche attraverso lo sviluppo di un sistema di proprietà industriale improntato alla sostenibilità. L'investimento da parte delle imprese in ricerca e sviluppo rappresenta il necessario punto di partenza, per avvicinarsi sempre di più ad un modello di business responsabile che presti attenzione alle esigenze e ai limiti dell'ambiente in cui viviamo. 
Le imprese italiane, nel corso dell'ultimo decennio, hanno dimostrato sempre più attenzione all'ambiente e alle nuove tecnologie innovative che ne permettono la preservazione. La dimostrazione di questa crescente focalizzazione è confermata anche dall'aumento significativo di deposito di brevetti per induzioni industriali cosiddetti "green", in quanto aventi ad oggetto:

- la produzione e lo stoccaggio di energie da fonti alternative;

- la gestione dei rifiuti;

- i materiali innovativi o alternativi volti a sostituire i materiali derivanti dal petrolio.

In Italia, le invenzioni "eco" risultano essere in media il 9,6\% del totale dei brevetti italiani depositati negli ultimi dieci anni, in linea con le percentuali a livello globale di depositi di brevetti "green" (Anonimo, 2020).

Tuttavia, affinché l'idea di sostenibilità e di circolarità non resti solo un'idea, appare necessario che questo approccio diventi sempre più generalizzato e condiviso, non solo da alcuni imprenditori "visionari" ma anche dall'intera filiera. Appare quindi determinante il ruolo dello Stato nell'incentivare le imprese a rendersi sempre più innovative, mettendo a disposizione delle stesse le risorse necessarie non solo in termini finanziari, ma anche in termini di collaborazione attraverso il mondo della ricerca universitaria.

In Italia, con la legge di bilancio 2021, è stato prorogato il Piano Nazionale Transizione 4.0 (introdotto dalla legge di Bilancio 2020), che rappresenta l'indirizzo della politica industriale in Italia, estendendo fino al 31 dicembre 2022 il supporto agli investimenti delle imprese in forma di crediti di imposta per determinate categorie di beni ${ }^{3}$. L'obiettivo del Piano Nazionale di Transizione 4.0, che con circa 24 miliardi di euro rappresenta il "primo mattone del Recovery Fund"4 e diventa una misura strutturale, è quello di stimolare gli investimenti privati, così da supportare le imprese nel processo di transizione tecnologica e di sostenibilità ambientale, riprendendo il ciclo di investimenti che si era arrestato a causa dell'emergenza sanitaria.

In ambito di innovazione, il Piano di Transizione 4.0 prevede la possibilità per le imprese che svolgono le seguenti attività di investimento in ambito di innovazione di diverse forme di supporto in forma di credito di imposta, in particolare:

- Ricerca e sviluppo, nello specifico attività di ricerca fondamentale, di ricerca industriale e sviluppo sperimentale in campo scientifico o tecnologico.

- Attività di innovazione tecnologica, diverse dalla ricerca e sviluppo di cui sopra, finalizzate alla realizzazione di prodotti o processi di produzione nuovi che si differenzino, rispetto a quelli già realizzati o applicati dall'impresa, sul piano delle caratteristiche tecnologiche o delle prestazioni o dell'eco-compatibilità o dell'ergonomia o per altri elementi sostanziali rilevanti nei diversi settori produttivi.

- Attività di innovazione green e digitale, inclusa attività tecnologica volta alla realizzazione di prodotti o processi di produzione nuovi o sostanzialmente migliorati per il raggiungimento di un obiettivo di transizione ecologica o di innovazione digitale. 
- Attività di design e ideazione estetica svolte da imprese operanti nel settore tessile e della moda, calzaturiero, orafo, dell'occhialeria, dell'arredamento e della ceramica per la realizzazione di prodotti nuovi a campionari.

\section{Sostenibilità sociale}

Per sostenibilità sociale si intende la capacità di garantire il benessere degli individui in ambito di sicurezza, salute, istruzione. Nel settore della moda, il concetto di sostenibilità sociale si traduce, secondo il Manifesto, nei seguenti comportamenti “virtuosi”:

- Rispetto dei diritti fondamentali dei lavoratori e tutela della loro sicurezza e salute sul lavoro.

- Corretta remunerazione e riconoscimento del valore contributivo degli stessi ai risultati dell'azienda.

- Valorizzazione del territorio, specialmente attraverso il sostegno dell'artigianato locale e del "Made in Italy".

- Contribuzione all'istruzione e alla formazione continua, nonché alla promozione dei giovani designer e delle start up.

- Favorire condizioni per lo sviluppo dei talenti e riconoscimento del merito, per tutti coloro che operano nell'azienda, valorizzando le pari opportunità e il giusto equilibrio tra vita privata e lavoro.

- Selezione dei fornitori, soprattutto se localizzati in Paesi in via di sviluppo, in base alla loro capacità di applicare e mantenere adeguati standard in termini di orari, remunerazione del lavoro, condizioni di salute e di sicurezza per i lavoratori.

Con particolare riferimento alla selezione dei fornitori, al fine di assicurare che l'intera filiera sia sostenibile, è di fondamentale importanza che i grandi brand si assicurino che i propri fornitori rispettino determinati standard di sostenibilità sociale, attraverso la previsione di apposite dichiarazioni e garanzie contrattuali.

Sempre con l'obiettivo di promuovere la sostenibilità sociale nell'industria della moda, la stessa CNMI ha recentemente pubblicato il "Social Sustainability Report" (Camera, 2020), dove in collaborazione con l'organizzazione indipendente Fair Wage Network ha condotto un sondaggio volto ad analizzare il contesto delle condizioni di lavoro e retributive all'interno della supply chain della filiera della moda e del lusso italiana.

Sono state raccolte le testimonianze di 45 fornitori e oltre 1.100 dipendenti, rappresentativi non solo delle diverse regioni italiane, ma anche delle varie specializzazioni in ambito di produzione, nonché delle diverse dimensioni aziendali (dalle fabbriche con centinaia di dipendenti alle piccole imprese a conduzione famigliare).

Il quadro che emerge della filiera del "fashion luxury" italiano, è un quadro generalmente positivo, grazie anche al fatto che tutte le imprese visitate applichino i contratti nazionali collettivi assicurando retribuzioni adeguate, oltre a politiche salariali premianti. 
Tutti i lavoratori intervistati hanno dichiarato di essere regolarmente remunerati, senza ritardi, e circa l' $81 \%$ ha dichiarato di essere completamente o parzialmente soddisfatto delle proprie condizioni di lavoro, mentre l' $82 \%$ ha dichiarato di essere completamente o parzialmente soddisfatto del proprio stipendio. Dal sondaggio, risultano comunque margini di miglioramento, sia per quanto riguarda il pagamento del lavoro straordinario, che per la necessità di legare maggiormente il salario agli obiettivi raggiunti e alle competenze professionali, anche in modo da valorizzare il contributo apportato dagli stessi lavoratori. La vera sfida, tuttavia è anche quella di esportare i principi della sostenibilità sociale all'interno dell'intero settore della moda. Ed è con questo intento che CNMI ha delineato una roadmap (Camera, 2020) in cui ha illustrato i principi guida per il raggiungimento di una sostenibilità sociale nell'industria della moda. I principi guida sono i seguenti:

- La necessità che siano i grandi brand della moda ad essere i vettori del cambiamento.

- Assicurare la regolarità delle remunerazioni salariali.

- Garantire livelli retributivi sufficienti a coprire le necessità fondamentali della vita.

- Contrastare le disuguaglianze e le discriminazioni salariali.

- Promuovere modalità retributive virtuose che valorizzino l'esperienza professionale $\mathrm{e}$ le conoscenze tecnologiche.

- Garantire l'aggiornamento temporale degli stipendi previsti dal CCNL.

- Ricordare che un giusto sistema retributivo è la chiave per una sana gestione delle Risorse Umane.

- Privilegiare sempre modelli sostenibili.

- Valorizzare la comunicazione e il dialogo sociale.

- Impegnarsi a creare le condizioni per un miglioramento continuo.

\section{Sostenibilità economica e identificabilità dei prodotti sostenibili: il ruolo del marchio}

L'ultimo pilastro dello sviluppo sostenibile è la sostenibilità economica, che esprime la necessaria sintesi fra sostenibilità ambientale e sociale, rappresentandone la vera garanzia nel tempo. L'azienda deve saper coniugare efficacemente il proprio impegno sociale e verso l'ambiente, assicurandosi dei relativi impatti economici, ma soprattutto, trasformandone il costo in valore riconosciuto dagli utilizzatori e in ultima analisi, da tutti gli stakeholders. Tra i principi, promossi dal Manifesto, in ambito di sostenibilità economica:

- la comunicazione dell'impegno ambientale e sociale attraverso forme periodiche di rendicontazione (ad esempio il "bilancio di sostenibilità").

- La condivisione, diffusione e valorizzazione presso clienti, dipendenti, i collaboratori e tutti coloro che operano con l'azienda dei valori di sostenibilità.

- Partecipazione e diffusione di stili di consumo responsabili attraverso un impegno con partner istituzionali e collaborazioni con l'Università. 
La creazione del valore che è possibile raggiungere attraverso l'adozione di politiche virtuose di "sostenibilità" ha, di per sé, quindi la necessità di essere comunicata e riconosciuta dai consumatori.

In questo senso, i marchi rappresentano un mezzo fondamentale ad ausilio della sostenibilità, in quanto permettono al consumatore di avere una maggiore consapevolezza nel momento in cui effettuano degli acquisti. I marchi, infatti, oltre a costituire un segno idoneo a distinguere un'impresa, col tempo possono anche diventare agli occhi dei consumatori simbolo di qualità e di standard del prodotto o servizio che intendono acquistare. In quest'ottica uno degli strumenti più efficaci a disposizione delle aziende è rappresentato dai marchi di certificazione.

Mentre in molti Paesi il marchio di certificazione esiste già da anni, in Italia e nell'Unione Europea è una novità piuttosto recente. Infatti, a livello europeo il marchio di certificazione è disciplinato dall'art. 83 del Regolamento (UE) n. 1001/2017 ("Regolamento Marchi”), mentre in Italia è disciplinato dall'art. 11 bis del codice di proprietà industriale, introdotto dal decreto legislativo n. 15/2019.

Il marchio di certificazione UE e italiano svolgono sostanzialmente lo stesso ruolo. Si tratta infatti di un marchio il cui compito è quello di attestare la conformità dei prodotti e servizi contraddistinti a determinate caratteristiche (come può essere ad esempio la produzione attraverso processi sostenibili).

Il Regolamento Marchi descrive i marchi di certificazione come segni

Idonei a distinguere i prodotti o i servizi certificati dal titolare del marchio in relazione al materiale, al procedimento di fabbricazione dei prodotti o alla prestazione del servizio, alla qualità, alla precisione o ad altre caratteristiche, ad eccezione della provenienza geografica, da prodotti e servizi non certificati ${ }^{5}$.

I richiedenti di un marchio di certificazione sono tenuti a depositare insieme al marchio un regolamento d'uso a cui dovranno conformarsi tutti gli utilizzatori di detto marchio. I regolamenti d'uso diventano quindi l'essenza stessa del marchio di certificazione, che devono indicare in particolare:

- le caratteristiche dei prodotti e dei servizi da certificare;

- le condizioni che disciplinano l'uso del marchio di certificazione;

- i criteri di prova e supervisioni che devono essere applicati dal titolare del marchio di certificazione.

Il titolare del marchio di certificazione, il quale può essere indifferentemente una persona fisica o giuridica, tra cui istituzioni, autorità e organismi di diritto pubblica, si impegna quindi a certificare che gli operatori che utilizzeranno il proprio marchio di certificazione si conformino alle prescrizioni dei regolamenti d'uso ed effettua i relativi controlli di conformità sotto la propria responsabilità. Il titolare del marchio di certificazione ha chiaramente un obbligo di imparzialità rispetto ai produttori e fornitori che utilizzano tale marchio per certificare i propri prodotti e servizi e, proprio per assicurare il ruolo neutrale del titolare, quest'ultimo non può gestire un'attività che comporti la fornitura di prodotti 
e servizi del tipo certificato. In tal senso, il marchio di certificazione non ha la tradizionale funzione distintiva di distinguere l'origine di un prodotto/servizio da un'impresa, piuttosto che da un'altra, ma piuttosto la funzione di distinguere i beni certificati da quelli non certificati, cosicché da consentire al consumatore di scegliere determinati prodotti o servizi in base alla presenza delle caratteristiche e degli standard garantiti dalla certificazione. La mancata verifica da parte del titolare del marchio del rispetto del regolamento da parte di coloro che lo utilizzano, comporta la decadenza del marchio stesso.

In Italia, attualmente risultano depositati circa 40 marchi di certificazione atti a distingure i prodotti della classe merceologica 25 (ovvero, articoli di abbigliamento, scarpe, cappelleria), di cui solo 4 già registrati.

Tra questi, un esempio è l'iconico marchio di certificazione "Woolmark" di titolarità di I.W.S. Nominee Company Limited (“the Woolmark Company”). Attraverso il proprio marchio di certificazione, the Woolmark Company garantisce che i prodotti in lana contraddistinti dallo stesso abbiano superato specifici controlli e che siano quindi conformi agli standard e requisiti di qualità previsti dal loro programma di certificazione. Attraverso l'apposizione di questo marchio, il consumatore è in grado di percepire l'impegno e l'attenzione che l'azienda produttrice pone nel realizzare i suoi prodotti, riconoscendone quindi l'indiscutibile valore.

Un altro esempio è il marchio di certificazione "Fair trade" depositato a livello UE in diverse classi merceologiche rilevanti nell'ambito della moda, tra cui la classe 24 (relativa a tessuti e stoffe) e la classe 25 sopracitata. Fairtrade, organizzazione internazionale che si occupa di migliorare le condizioni degli agricoltori nei Paesi in via di sviluppo, certifica una varietà di prodotti fra cui anche il cotone. Tramite un marchio di certificazione "Fairtrade", un operatore del settore della moda può quindi garantire ai propri consumatori che il suo prodotto è stato realizzato nel rispetto dell'ambiente e della società.

Il sempre più diffuso utilizzo dei marchi di certificazione potrebbe quindi rappresentare da un lato un modo per trasmettere al consumatore una maggiore consapevolezza della storia e della qualità del prodotti e dall'altro anche un modo per spingere le aziende a conformarsi sempre di più a determinati standard, così da bilanciare i costi sostenuti per l'impletazione di modelli sostenibili in vero e proprio brand value percepibile dal consumatore finale e da tutti gli stakeholder.

\section{Investire in sostenibilità - Case Study}

Esistono già diversi casi di aziende italiane "virtuose" che sono state in grado di coniugare i tre pilastri della sostenibilità ottenendo modelli di business di successo. Degna di nota è ad esempio l'esperienza della Candiani S.p.A.

La Candiani S.p.A. (nota con l'insegna “Candiani Denim”) è un'azienda fondata nel 1938, che opera da sempre interamente in Italia, più precisamente a Robecchetto con Induno (MI). L'azienda è verticalmente integrata e la produzione avviene in due stabilimenti che coprono una superficie produttiva complessiva di 85.000 metri quadrati. Con quasi 700 operai e 23.5 milioni di metri lineari annui, la Candiani è la tessitura di denim più grande 
d'Europa. Nonostante spesso si citi la localizzazione in Italia come un fattore di svantaggio, la società presenta un bilancio economico estremamente positivo, annoverando fra i propri clienti i marchi più prestigiosi della moda e del premium denim industry come Lee, Levi's, and Lucky Brand, 3x1, Hugo Boss, and Mott \& Bow, o ancora J. Crew, Madewell, and Outerknown.

Nel “Sustainaibility Report 2019-2020" dell'azienda possiamo leggere:

Un tipico paio di jeans -realizzato dal cotone coltivato e fabbricato in India, cucito in Bangladesh e venduto nei Paesi Bassi- si dice possa avere dei costi ambientali e sociali nascosti di $€ 30$. Il risultato è un divario di prezzo, il che significa che molte paia di jeans nei negozi oggi sono grossolanamente sottostimati. L'esclusione dei costi per la società, come l'uso dell'acqua, l'inquinamento dell'aria, il cambiamento climatico e i bassi salari nei cartellini dei prezzi, pongono alcuni rischi che non possiamo più permetterci di ignorare (Candiami, 2020).

Le innovazioni che Candiani ha sviluppato, negli ultimi vent'anni, hanno permesso all'azienda di conseguire significative economie in tutte le fasi della produzione. Ad esempio, nella filatura, Candiani utilizza principalmente cotone, sia quello biologico, sia quello della Better Cotton Initiative (BCI), sia quello convenzionale che, infine, quello riciclato che la società recupera in loco dagli scarti di cotone. L'azienda utilizza anche tessuti derivati dal legno come Tencel, MicroModal e Refibra. Ugualmente innovativa nella tintura del filato, il quale viene sottoposto a un bagno di indaco Indigo Juice, che utilizza il $15 \%$ in meno di acqua e il $15 \%$ in meno di energia rispetto ai metodi convenzionali di tintura dell'indaco; oppure viene tinto con la tintura N-Denim, che riduce il numero di bagni di tintura da sette a due, e utilizza il 30\% in meno di prodotti chimici rispetto ai processi di tintura convenzionali; oppure viene tinto con Kitotex, che è realizzato con l'esoscheletro di gamberi di scarto (Candaini, 2020).

Un altro importante esempio sul panorama italiano è quello di Carmina Campus di Ilaria Venturini Fendi, figlia di Anna, una delle cinque sorelle Fendi. Il progetto nasce nel 2006 al fine di proporre un diverso approccio alla creatività fondato sulla rigenerazione dei materiali. Nello specifico, la strategia imprenditoriale di Carmina Campus è quella legata al riuso dei materiali di scarto, come l'alluminio, la gomma o i tessuti. Attraverso il lavoro di artigiani italiani vengono realizzate borse, accessori e mobili, tutti pezzi unici e tutti realizzati con l'utilizzo di materiale riciclato. Ulteriore elemento "sostenibile" è il fatto che tutti i capi realizzati non sono legati ad una specifica stagione, superando quindi l'idea di capi “usa e getta” (Gazzola y D’ll Alva, 2020).

Le attività di Carmina Campus non si limitano tuttavia solo alla realizzazione di borse e accessori ma si estendono anche a collaborazioni internazionali volte a esportare il proprio modello di business anche all'estero. In particolare, tramite la collaborazione con ITC -International Trade Centre- il progetto Carmina Campus è stato sviluppato anche in Camerun, coinvolgendo comunità rurali del Kenya e dell'Uganda L'obbiettivo di queste collaborazione è quello di esportare il know-how del progetto al fine di realizzare nuove opportunità di lavoro per le persona marginalizzate che attraverso i programmi di for- 
mazione offerti da ITC hanno acquisito le competenze professionali sufficienti per poter operare all'interno del progetto (Poletti, 2010).

Questi casi sono solo alcuni dei diversi esempi di eccellenza italiana in prima linea nella rivoluzione del settore della moda verso un sistema di produzione e consumo sostenibile, dimostrando che sostenibilità e strategie di business possono anche andare a pari passo.

\section{Conclusioni}

La crisi pandemica, pur nella sua gravità e negli oneri che sta causando a ogni segmento della società, rappresenta un fattore di cambiamento valoriale per i consumatori, che oggi più che mai sono alla ricerca di una dimensione più equilibrata o, appunto, "sostenibile". La stessa crisi sta imponendo, in Italia come in tutto il mondo, la necessità di ripensare il proprio modello di business e, come sempre, è nelle crisi più difficili che si celano le migliori opportunità.

La crescente consapevolezza nell'opinione pubblica, dei limiti della sostenibilità del sistema moda tradizionale, riflessa nella maggiore attenzione riservata dalle aziende, anche in forma associativa, all'adozione di modelli di business sempre più rispettosi dell'ambiente, è da considerarsi non come un vincolo ma come una straordinaria opportunità di innovazione e di creazione di nuovo valore.

In questo senso, la proprietà industriale gioca un ruolo chiave, da un lato, nella protezione di invenzioni industriali green, dall'altro nel contraddistinguere, attraverso i marchi di certificazione, i prodotti e i servizi che posseggono caratteristiche di sostenibilità.

Come confermato dalla casistica analizzata, le aziende italiane della moda che meglio hanno interpretato questa nuova sensibilità dei consumatori, anticipandola a volte e rendendola parte integrante e fondante dei propri valori e delle proprie strategie, sono riuscite a competere efficacemente e con successo su scala globale.

\section{Notas}

1. Visitato il 15 gennaio 2021.

2. Le imprese e le organizzazioni che hanno preso parte ai lavori di predisposizione del documento sono state: Ermenegildo Zegna, Salvatore Ferragamo, Guccio Gucci, Yoox, Limonta, Taroni, Simonetta, Material Connexion, Sistema Moda Italia, Politecnico di Milano, Università Bocconi di Milano, Avanzi - Sostenibilità per Azioni. Le indicazioni contenute nel Decalogo sono comunque unicamente riferibili a Camera Nazionale della Moda Italiana.

3. https://www.agendadigitale.eu/industry-4-0/piano-transizione-4-0-il-nuovo-credito -di-imposta-tutte-le-aliquote-e-i-beni-compensabili/

4. https://www.mise.gov.it/index.php/it/transizione40 
5. A differenza del marchio di certificazione europeo, in base all'art. 11 bis del codice di proprietà industriale italiano, il marchio di certificazione nazionale può essere utilizzato anche per indicare l'origine geografica di un prodotto e servizio.

\section{Referencias bibliográficas}

Anonimo (21 de diciembre de 2020). Green technologies: l'analisi UIBM sui dati dei brevetti presentati nel campo delle tecnologie eco-sostenibili. Recuperado de 21 dicembre 2020. https://sistemaproprietaintellettuale.it/notizie/brevetti/20105-green-technologies. html?title=green $\% 20$ technologies .

BOF Studio. (5 de diciembre de 2020). CNMI launches Report and Roadmap to Socially Sustainable Wages Practices in Fashion, The Business of Fashion. Recuperado de: https:// www.businessoffashion.com/articles/sustainability/cnmi-launches-report-and-roadmap -to-socially-sustainable-wage-practices-in-fashion.

Business for Social Responsibility (19 de julio de 2018). Disrupting Luxury: Creating Resilient Business in Times of Rapid Change. Recuperado de: https://www.bsr.org/en/ our-insights/report-view/disrupting-luxury-creating-resilient-businesses-in-times-of-rapid-change

Camera Nazionale della Moda Italiana - Fair Wage Organization. (15 de diciembre de 2020) Social Sustainability Road Map. Recuperado de: https://drive.google.com/file/d/ 15gw6ImwAQ48tvacOkuWVEdPMD3-7484d/view

Camera Nazionale della Moda Italiana (13 de junio de 2012). Manifesto della Sostenibilità per la moda italiana. Milano. Recuperado de: https://www.cameramoda.it/media/docu ments/roundtable/manifesto/manifesto_sostenibilita_it.pdf

Camera Nazionale della Moda Italiana (15 de diciembre de 2020). Social Sustainability Report. Recuperado de https://drive.google.com/file/d/18g8tjBwAYcWj0Bqv6fp62fUH Vx_xJWn1/view

Candiani Denim (2020), Sustainability Report 2018-2019, https://www.greenmatters.com/p/ candiani-sustainable-denim

Comisión Europea (s.f.). Textiles and clothes in the EU. Recuperado de: https://ec.europa. eu/growth/sectors/fashion/textiles-clothing/eu_en

Ellen McArthur Foundation (28 de noviembre de 2017), Report: A New Textiles Economy: Redesigning Fashion's future. Recuperado de: https://www.ellenmacarthurfoundation. org/publications/a-new-textiles-economy-redesigning-fashions-future.

Gazzola, P.; Pavione, E. y Dall'Ava, M. (febrero, 2020). I differenti significati di sostenibilità per le aziende del lusso. Economia Aziendale Online - Business and Management Sciences Quarterly Review Vol. 10.4/2019. Pavia, Italia.

Guercini, S. y Ranfagni, S. (2013). Sustainability and Luxury: The Italian Case of a Supply Chain Based on Native Wools. The Journal of Corporate Citizenship, no. 52. pp. 76-89. Recuperado de: JSTOR, www.jstor.org/stable/jcorpciti.52.76. Accessed 15 Jan. 2021.

Hirsh, S. (26 de noviembre de 2019). Cooking up sustainable jeans with Candiani. Recuperado de: https://www.greenmatters.com/p/candiani-sustainable-denim 
Mancarella, M. (14 de noviembre de 2006). Il principio dello sviluppo sostenibile: tra politiche mondiali, diritto internazionale e Costituzioni Nazionali. Giuristi Ambientali. Parlamento Europeo (3 de marzo de 2021). The impact of textile production and waste on the enviroment (inforgraphic). Recuperado de: https://www.europarl.europa.eu/news/ en/headlines/society/20201208STO93327/the-impact-of-textile-production-and-waste -on-the-environment-infographic

Poletti, F. (20 de julio de 2010). Carmina Campus. Vogue Italia. Recuperado de: https:// www.vogue.it/talents/nuovi-talenti/2010/07/carmina-campus

Tartaglione, C.; Gallante, F. y Guazzo, G. (julio 2020) SOSTENIBILITÀ: MODA Cosa significa, come si applica dove sta andando l'idea di sostenibilità nel sistema moda. Recuperado de: https://informatex.it/wp-content/uploads/2017/06/Sostenibilit\%C3\%A0-Moda.pdf

World Commission on Environment and Development (1987). Our Common Future. Recuperado de: https://sustainabledevelopment.un.org/content/documents/5987our-co mmon-future.pdf

Young, S. (23 de abril de 2020) Fashion Revolution Week: What Was The Rana Plaza Disaster And Why Did It Happen? Independent. https://www.independent.co.uk/life-style/ fashion/rana-plaza-factory-disaster-anniversary-what-happened-fashion-a9478126.html

\begin{abstract}
The current pandemic is shifting consumers' attention towards the adoption of a more sustainable attitude in their consumption choices. Lacking of a specific international and domestic regulation concerning sustainability, it is often the same companies, or companies associations, such as the Italian National Chamber of Fashion, to adopt self-regulations to ensure the development of a more sustainable business model. In this respect, intellectual property is a key to distinguish a sustainable business, on the one hand for the research and development activities that lead to the filing of green patents; on the other, through the identification of sustainable features of products and services identified by certification marks.
\end{abstract}

Keywords: sustainability - consumption - business model - green patents - certification marks - intellectual property - consumers - research and development.

Resumo: A atual pandemia está mudando a atenção dos consumidores para a adoção de uma atitude mais sustentável em suas escolhas de consumo. Na falta de uma regulamentação nacional e internacional específica sobre a sustentabilidade, muitas vezes são as mesmas empresas, ou associações de empresas, como a Câmara Nacional da Moda da Itália, que adotam autorregulamentações para garantir o desenvolvimento de um modelo de negócios mais sustentável. Nesse sentido, a propriedade intelectual é a chave para distinguir um negócio sustentável, por um lado, para as atividades de pesquisa e desenvolvimento que levam ao depósito de patentes verdes; de outro, por meio da identificação de características sustentáveis de produtos e serviços identificados por marcas de certificação. 
Palavras chave: sustentabilidade - consumo - modelo de negócios - patentes verdes - marcas de certificação - propriedade intelectual.

[Las traducciones de los abstracts fueron supervisadas por el autor de cada artículo] 